\title{
Discussion on the tumor treatment of traditional chinese medicine: Discover the new therapeutic strategy
}

\author{
Xiaojuan Sun* and Fucheng $\mathrm{Hu}$ \\ Shenzhen Tumor Immune-gene Therapy Clinical Application Engineering Lab, Biobank of the Second People's Hospital, The First Affiliated Hospital of Shenzhen \\ University, Shenzhen, China
}

\begin{abstract}
This review introduced the tumor treatment of traditional Chinese Medicine, discussed its holistic view of treatment compare to approaches of targeting tumor cells. Put forward the point that restoration of the energy of patients is the main strategy for tumor therapy.
\end{abstract}

More evidence shows that tumor incidence increases annually worldwide. A majority of advanced cases tend to treatment failure [1].

Most of the western medicine doctors suppose that the tumor should be removed in order to prevent the illness further development. Therefore the surgery, radiation, and chemotherapy are applied universally in the tumor treatment based on the understanding. However, some severe side effect is often very serious. Patients undergoing therapy showed symptoms of sickness, nausea, trichomadesis, and poor quality of life. The physical condition of patients often declined instead of getting the anticipated result after completing the full course treatment [2].

Compare to western medicine, Chinese medicine consider the root cause of tumorigenesis is decline of the immunity due to energy deficiency [3].The treatment should not be confined to removing the tumor cells alone, but instead boost the body energy levels, enhance the immunity, which is the most effective therapy [4]. Specifically, "YinYang" imbalance and disequilibrium occurs in tumor patients, the weakness of "Yang" is universal in patients, which suggests blockage of the normal energy metabolism, diminished or even stagnant energy status. To some extent, tumor formation is largely due to long-term stasis of the local metabolites, the stasis leads to the blockade of local meridians, impedes normal energy flow of the network, accordingly entering a vicious cycle. The famous ancient Chinese Medicine Zhongjing Zhang put forward the "cold" is the major cause of most diseases, referred to as deficiency of "Yang." Based on the therapeutic principles of "Yin-Yang balance", improving "Yang" and dispelling "cold" are the main therapeutic regimen. Aim of fostering "Yang" is emphasized [5]. The treatment methods are diversity, including Chinese herbs, acupuncture and moxibustion. In the typical prescriptions, Radix aconiti carmichaeli and Ginger are the two most used presentative herbs. Many reports show their remarkable effects on strengthening and activating the "Yang"-energy in the treatment of lung and breast cancers [6,7]. The acupuncture is another important way to balance "Yin-Yang", mainly through dredging Meridians and reconciling metabolism as well as providing significant pain relief [8]. Besides, there are some complementary therapy such as skin scraping, massage, topical ointments and cupping, which are all effective, natural and safe physical therapy.

Actually, some scientists engaged in the Western medicine already recognized that tumor is caused by the whole systemic imbalance. In 2004 and 2005, Academician of China Gu J.R. and Yang S.L. published reports in the Proceedings of the National Academy of Sciences (PNAS) and Chinese Medical Journal, pointing out that tumor is a kind of disease characterized by abnormal growth of local tissues with general systemic deregulation $[9,10]$. Wu M.C, the reputed Chinese hepatobiliary surgeon, put forward that hepatic carcinoma is a general systemic disease; the pathological changes in liver are only local performance. He proposed combining the Chinese and Western medicine for the better therapeutic outcome, as Chinese medicine more emphasizes the holistic approach [11]. In the opinion of Chinese medicine, the patient is not considered as cured after tumor removed but only when the patients' energy levels are restored, spiritually healed, and without any remnants of pathology. Even though the tumor resection is succeed, the symptoms is disappeared, the deficiency of "Yang" or energy still can leads to tumor relapse.

Another important view of Chinese medicine is that "the illness comes from the mind". Our brain is like the command center of body, manage the operation of whole human body system and provide us a healthy state. Keeping the appropriate emotion is crucial for getting the best work efficiency of brain [12]. A study published in Cell in $2010[13,14]$, reported the role of brain in tumor development. In this study, researchers placed a group of mice with tumors in an enriched environment (EE) with a variety of toys in the cage. Compared with the control mice, EE significantly reduced the tumor burden in the model mice of syngeneic melanoma and colon cancer. In addition, it suggested that the EE paradigm activated the hypothalamus, increased the expression of brain-derived neurotrophic factor (BNDF), leading to preferential activation of sympathetic innervation of white adipose tissue, which in turn led to suppression of leptin expression and release via beta adrenergic receptors, decreased leptin-inhibited tumor growth. This suggested that the mental activity have a great impact on tumor

Correspondence to: Dr. Xiaojuan Sun, Shenzhen Second People's Hospital the First Affiliated Hospital of Shenzhen University, 3002 West Sungang Road, Shenzhen, 518035, China, Tel: +0086 0755-83366388, Fax: +0086 075583356952 , E-mail: xiaojuan26@gmail.com

Received: March 28 2016; Accepted: April 25, 2016; Published: April 29, 2016 
development. Gu J.R. highlighted a three-pronged perspective in his " $1 / 3$ " opinion: " $1 / 3$ " of patients die of natural tumor development trend, " $1 / 3$ " of patients die of over-treatment, and " $1 / 3$ " of patients die of fear and depression. He told the patients, "Be happy, tumor may stay away from you".

We may take tumor cells as defective products generated by body due to energy deficiency (Figure 1). Usually, anxiety, depression and angry etc. negative aspects cause huge consumption of energy, while happiness, tolerance and calm etc. positive thinking produce amount of energy. In the meanwhile, unhealthy diet causes increasing body fluid acidity, accumulation of waste, disruption of normal metabolism, leading to susceptible to chronic diseases [15]. On the other hand, environmental pollution, rotten soil or water, and abuse of pesticides and fertilizers, the essential nutrients are seriously depletion in the food and drinking water. The loss of nutritional also results in decrease of restore function of cells when cells damage and dysfunction [16]. The study by Nobel Prize winner M.Bishop and H.Varmus showed that the oxygen radicals are the main culprit in chemically induced carcinogenesis [17]. The normal genes in cells are converted into oncogenes by chemical carcinogens, which contribute to the abnormal cell proliferation [18].

Energy supplementation is required to improve the physical condition, enhance immunity, and support the "Yang" of body. Energy deficiency can be caused by excessive energy (E) consumption by brain, and insufficient Calorie (Q) supplement from food. (Figure 2). The energy $\mathrm{Q}$ is mainly produced by oxidation of food. Although we know balanced nutrition should be proportionate and comprehensive, the dietary concept and structure changed significantly according to one's taste. People tend to intake of excessive high calorie food, such as meat, desserts, soft drink today, which generate a lot of acidic metabolic products in the body, besides, the body need additional energy for their metabolism. More importantly, the excessive intake increases the oxidative metabolites (mo) and oxygen radicals $\left(\mathrm{O}_{2}^{-}\right)$.

The primary purpose of food intake is to generate energy via aerobic respiration, as represented by the following equation:

\section{$\mathrm{M}+\mathrm{O}_{2} \underset{\text { Enzyme }}{\stackrel{\mathrm{H}_{2} \mathrm{O}, 36.5^{\circ} \mathrm{C}}{\longrightarrow}} \mathrm{CO}_{2}+\mathrm{mo}^{+}+\mathrm{O}_{2}^{-}+\mathrm{Q}$}

When the products on the right side of the equation are not promptly removed, the oxidation speed is slow, causing reduced energy production which affects the normal body metabolism. At the

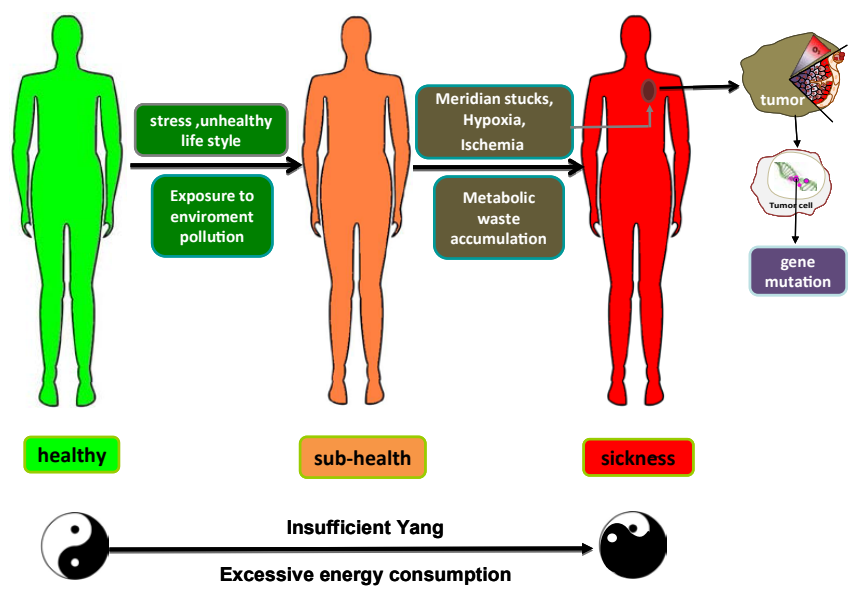

Figure 1. Energy deficiency leads to the formation of tumor.

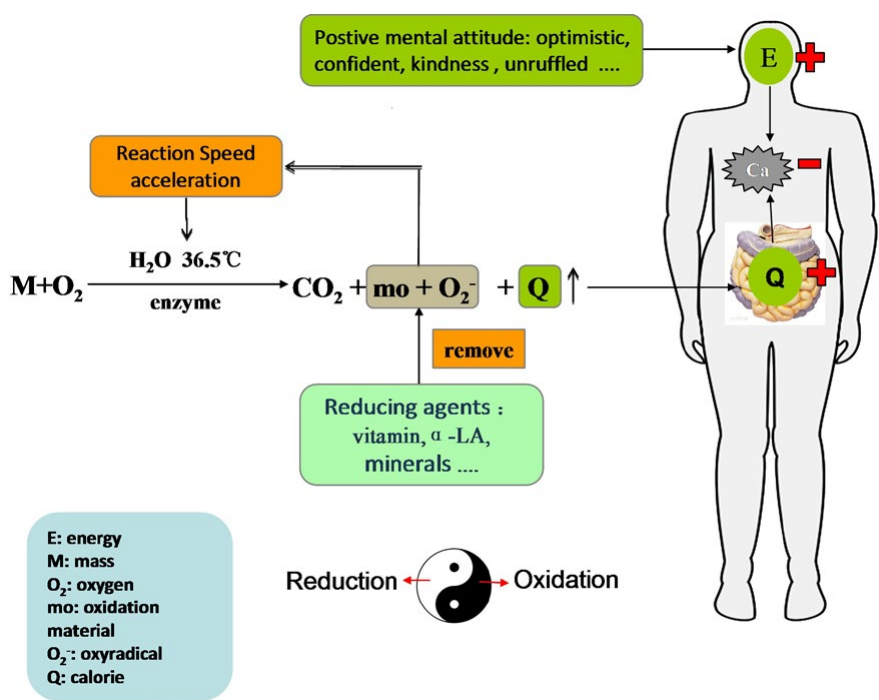

Figure 2. Restore the energy is the main strategy in tumor therapy.

same time, the high level of metabolites and oxygen radicals causes cellular and molecular damage. Therefore, we call the "over oxidization and insufficient reduction" is a common metabolic problem for most of people. The corresponding approach is to supplement body with a large number of electronating agent, including vitamins, minerals, phytochemicals and other natural antioxidants, which are essential to clear excessive oxygen redicals in human body $[19,20]$.

Chinese medicine take much count of the utilization of food on the body function, which is the foundation of the acquired constitution human body, as the processes produce the energy to support all metabolism activities [21]. "Food, pierce, acupuncture, moxibustion and medicine" are the five main traditional treatment methods, in which food ranks first. In the case of insufficient energy, the patient recovery is greatly delayed. Hippocrates said, " let the food be the medicine and the medicine be the food" [22]. So through the modern nutriology, the Chinese medicine may be understand by the Western medicine and applied in the worldwide. As the view of holism and balance, the therapeutic way is a process of body restoration rather than overtreatment is worthy to be studied.

In our opinion, the proper tumor therapy constitutes two parts: "autonomous therapy" and "supportive therapy". The former mainly relies on patients' understanding of disease and self-management, including stopping the inappropriate thoughts and incorrect lifestyle, such as limited exercise, late-night activity, overwork and unhealthy dietary habits. Correcting the negative habits or psychology plays a key role in reducing energy $\mathrm{E}$ consumption, may affect $70 \%$ of the therapeutic effect. Just as the old saying, "illness comes or goes dependent on the mind". The "supportive therapy" is the methods aim to help patients with better life plan as diet, rest, exercise, dredging the main and collateral channels and improve the energy Q. The good effect can only be attained though the two approaches by active both of doctors and patients effort. Particularly, it should be mentioned that the patient is play the leading role in a doctor-patient relationship and the whole process of therapy, as doctors cannot substitute for patients' efforts in altering their own psychological condition.

It is notable, the concept of "participatory medicine" representing patients' thoughts has been paid attention now [23]. It refers to spiritual guidance for patients and doctor-patient interactions. In addition to 
focusing on tumor itself, it's necessary to engage the patient physically and mentally in the treatment. The misconceptions or improper practice by some clinicians who are obsessed with the disease itself more than the patient may need be corrected [24].

\section{References}

1. Chen JG, Lu JH (2007) Present situation of cancer prevention. Oncology 27: 755-759.

2. Luo J, Sun Y (2001) Quality of life in cancer patients. Chinese Journal of Oncology 10: 76-78.

3. Cheng DH (2010) Understanding and prevention of cancer etiology in traditional Chinese medicine. Journal of Changchun University of Chinese Medicine 26: 511-512.

4. Zhang YH, Ye LJ, Peng HY (2009) Treatment of deficiency and toxicity for cancer. Journal of NanjingUniversity of Traditional Chinese Medicine 25: 408-411.

5. Zhang QY (2006) A treatment principle of protecting vital-energy in Treatise on Febrile Diseases. JilinJournal of Traditional Chinese Medicine 26: 3-5.

6. Wang CC, Jia WK (2014) Experience of lung cancer treatment by Professor JiaWenkui. World Journal of Integrated Traditional and Western Medicine 9: 467-469.

7. Zhang WW (2014) Treatment of 62 cases suffering from upper limb lymphedema after mastectomy of breast cancer with activity yang and promoting diuresis. Jiangsu Journal of Traditional ChineseMedicine 2: 019.

8. Chen ZJ, Guo YP, Wu ZC (2008) [Observation on the therapeutic effect of acupuncture at pain points on cancer pain]. Zhongguo Zhen Jiu 28: 251-253.[Crossref]

9. Wan D, Gong Y, Qin W, Zhang P, Li J,et al. (2004) Large-scale cDNA transfection screening for genes related to cancer development and progression. Proc Natl Acad Sci U S A 101: 15724-15729..[Crossref]

10. Gu JR, Yang SL (2005) Cancer with a new concept of systemic disease. Chinese Medical Journal 85: 505-507.

11. Wu MC (2003) [Traditional Chinese medicine in prevention and treatment of liver cancer: function, status and existed problems]. Zhong Xi Yi Jie He Xue Bao 1: 163-164. [Crossref]
12. Wen CL (2014) Tapping gold stirrup and heavy saddle- Review of "three books for health preserving" by Professor Li PW.Family \& Traditional Chinese Medicine: 78-79.

13. Cao L, Liu X, Lin EJ, Wang C, Choi EY, et al. (2010) Environmental and genetic activation of a brain-adipocyte BDNF/leptin axis causes cancer remission and inhibition. Cell142: 52-64.[Crossref]

14. Li G, Gan Y, Fan Y, Wu Y, Lin H,et al. (2015) Enriched Environment Inhibits Mouse Pancreatic Cancer Growth and Down-regulates the Expression of Mitochondria-related Genes in Cancer Cells. Sci Rep 5: 7856. [Crossref]

15. Zhou G., Jiang L.P. and Fan C.W., et al. Acidic Physique and sub-health state. Journal of ChineseModern Medicine 4: 815-816 (2007).

16. Valko M, Leibfritz D, Moncol J, Cronin MT, Mazur M, et al. (2007) Free radicals and antioxidants in normal physiological functions and human disease. Int J Biochem Cell Biol 39: 44-84.[Crossref]

17. (2011) Modern new concept of nutrition (health gospel for busy and lazy people) [M] Chinese Social Press.

18. Valko M, Izakovic M, Mazur M, Rhodes CJ, Telser J (2004) Role of oxygen radicals in DNA damage and cancer incidence. Mol Cell Biochem 266: 37-56.[Crossref]

19. Vaya J, Aviram, M (2001) Nutritional antioxidants mechanisms of action, analyses of activities and medical applications. Current Medicinal Chemistry-Immunology, Endocrine \& Metabolic Agents 1: 99-117.

20. Podsedek A (2007) Natural antioxidants and antioxidant capacity of Brassica vegetables: A review. LWT-Food Science and Technology 40: 1-11.

21. Li Z,Chen YB (1999) Progress of relationship between splenasthenic syndrome and immunity in traditional Chinese medicine. Journal of Snake 11: 43-45.

22. Ullah MF,Khan MW (2008) Food as Medicine: Potential Therapeutic Tendencie of Plant Derived Polyphenolic Compounds. Asian Pac J Cancer Prev 9: 187-196. [Crossref]

23. Bao SS,Shi XY (2012) Construction of a harmonious doctor-patient relationship with the concept of 4P. Soft Science of Health 26: 954-955.

24. Yang GT, He XS,Li Z (2006) Contemporary medical technology development and humanistic care. Medicine and Society 19: 36-37.

Copyright: (C) 2016 Sun X. This is an open-access article distributed under the terms of the Creative Commons Attribution License, which permits unrestricted use, distribution, and reproduction in any medium, provided the original author and source are credited. 